\title{
Radar Plane Position Indicator Fast Debugging Equipment Based on USB Data Acquisition Technique
}

\author{
Wang Qiang ${ }^{1}$,Zhang Xuezhi ${ }^{1}$, Tang Xiaokang ${ }^{1}$, Xie Cun $^{1}$, Ma Yongshan $^{2}$ \\ ${ }^{1}$.Wuhan Mechanical Technology College, Wuhan, China \\ ${ }^{2}$ The 65370 Department, Changchun, China
}

\begin{abstract}
This paper introduces the design of a radar plane position indicator (PPI) fast debugging equipment based on USB. Hardware platform is a high-speed 16 channel date acquisition card -USB4716, which is based on USB. Its internal actual is 8 parallel 16AD module. The sampling length and sampling frequency can be set by software. PC interface compile by VC++2008, and finish data processing and waveform.
\end{abstract}

Introduction: The radar PPI fast debugging equipment is a dedicated device for fast precise debug of the radar PPI by monitoring the output signals of the PPI display control circuit and comparing them with the standard signals. Traditionally, the radar PPI is debugged with the calibration system built of discrete instruments. This method is denounced for long debugging period, large space occupation, complex wiring, susceptibility to disturbance and difficult operation. The device proposed in this paper adopts the USB data acquisition technique and has the merits of fast debugging speed, high calibrating precision, small space occupation, robustness to disturbances, easy operation and high extensibility.

\section{Top Design Of The Debugging Device}

The top architecture of the radar PPI fast debugging device is displayed in Figure 1. As is shown, the debugging device is composed of a signal generation module, a data acquisition and process module and a display module. The debugging device acquires the output signals of the PPI display control circuit and stores the data in the micro control unit (MCU) module. After the data is analyzed in the MCU, the process result is displayed on the screen in a form similar to the radar PPI, and the debugging plan can be produced and provided to the users.

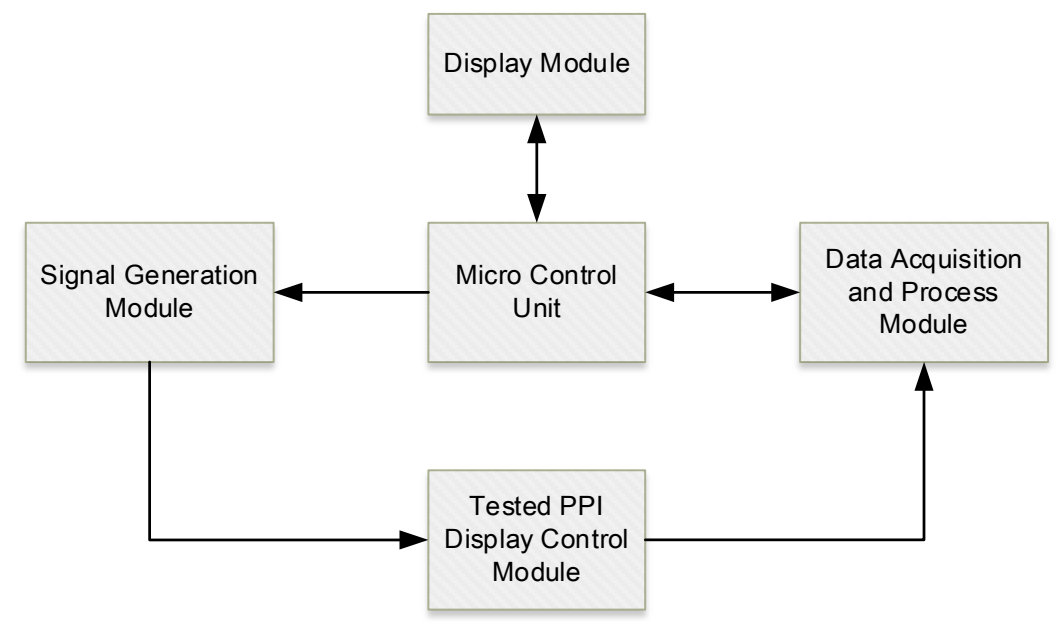

Figure 1 The top architecture of the radar PPI fast debugging device

\section{USB-4716 Data Acquisition Card}

USB-4716 is a plug-and-play data acquisition device, which provides 16 single-ended or 8 differential analog input channels at a 16-bit resolution and a sample rate up to $200 \mathrm{k} / \mathrm{s}, 16$ digital I/O 
channels, a 32-bit counter and two 16-bit analog output channels. USB-4716 is powered by the USB interface and can operates without the need of extra power supplies. The schematic of USB-4716 is shown in Figure 2.

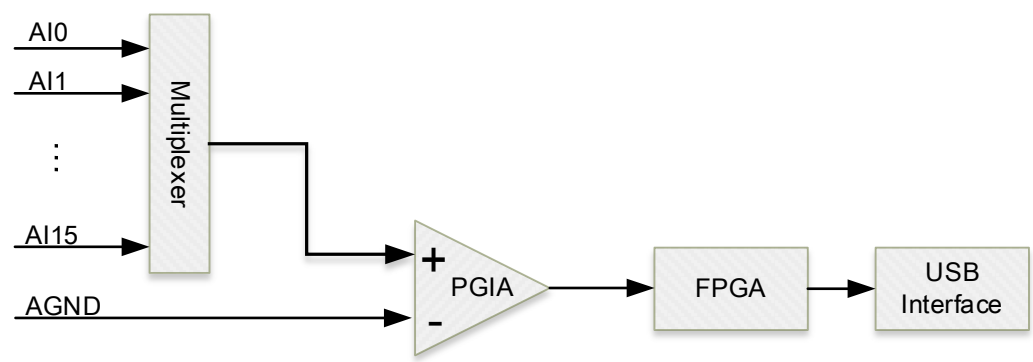

Figure 2 The schematic of USB-4716

\section{Design Of The Data Acquisition And Process Module}

Real-Time Acquisition Of Analog Signals.The software for real-time data acquisition was developed on the platform of $\mathrm{VC}++2008$. Owing to the dynamic link library and interface functions provided with USB-4716, the development process was quite convenient. The detailed development steps are as follows:

Firstly, copy compatibility.h, inc/bdaqctrl.h to the project folder and add them to the VC++ project. Then initialize the variables of the project.

The simplified codes are shown as follows:

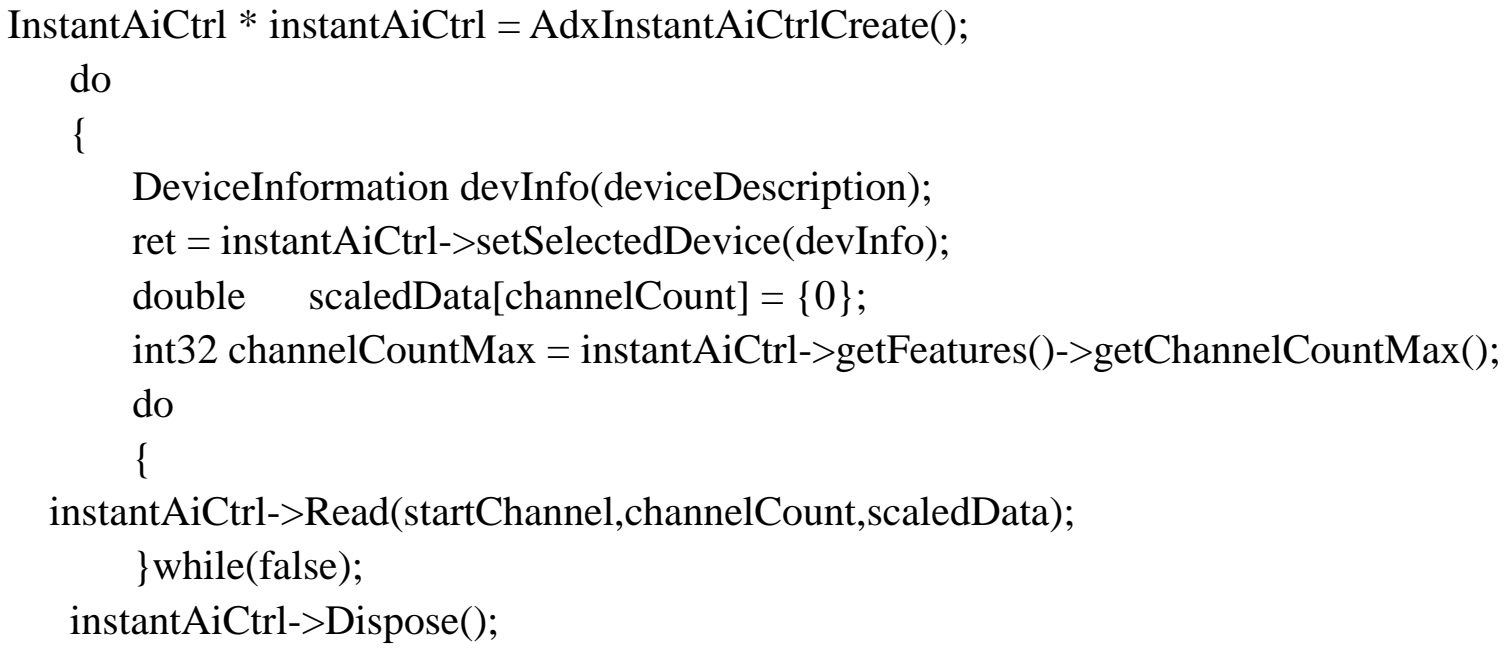

where startChannel is first data acquisition channel, channelCount is total number of data acquisition channels. The operation procedure adopted by the data acquisition software of the debugging device is shown in Figure 3. 


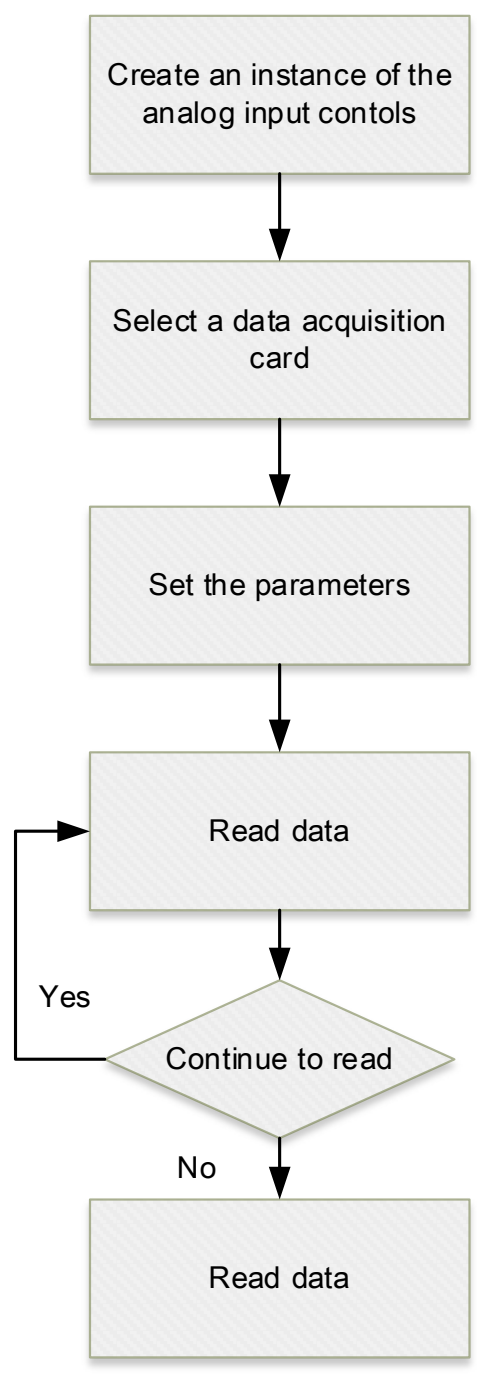

Figure 3 The designed operation procedure of the data acquisition software

Improved Peak-To-Peak Value Computation Algorithm.An improved peak-to-peak value computation algorithm was proposed to precisely obtain the peak-to-peak values of the output signals of the PPI display control circuit and filter the disturbance signals introduced in the data acquisition procedure. The conventional peak-to-peak value computation algorithm traverses the acquired data and compare them. If one sample data is lager or less than both its former and latter neighbors, this specified data is considered extreme point. Then the average of all the extreme points is calculated as the peak-to-peak value. Nevertheless, in actual experiments, the peak-to-peak value obtained with this method deviated much from its actual value. In fact, with the existence of disturbance signals, the conventional peak-to-peak value computation algorithm will recognize fake extreme points and thus produces a great computation error. In the improved peak-to-peak value computation algorithm, some special processes are introduced to solve this problem. The preliminary extreme points are compared with a preset threshold, and only those greater than the threshold are left and used to compute the peak-to-peak value. The detailed codes are as follows:

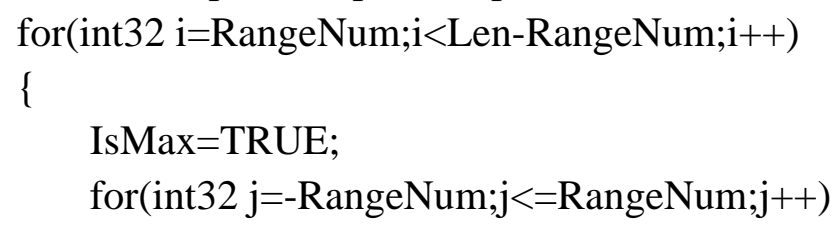




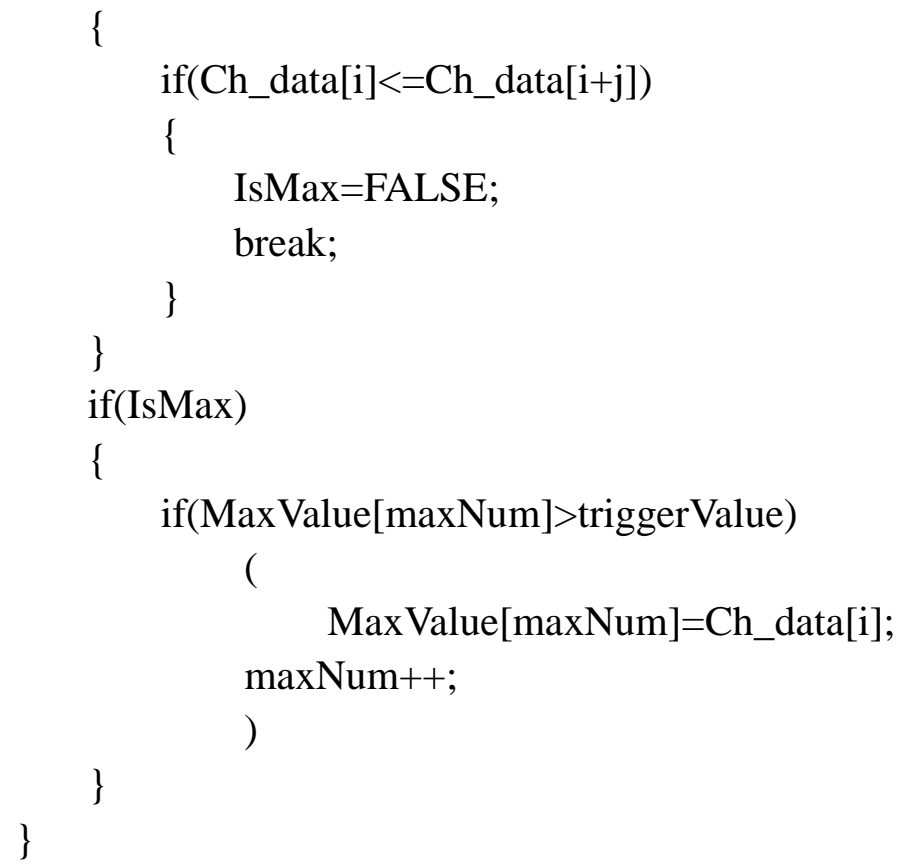

The extreme points after process are stored in the array MaxValue[maxNum] and averaged. The final result is displayed with scanning lines. The waveform and the fianl debugging plan are shown in Figure 4.

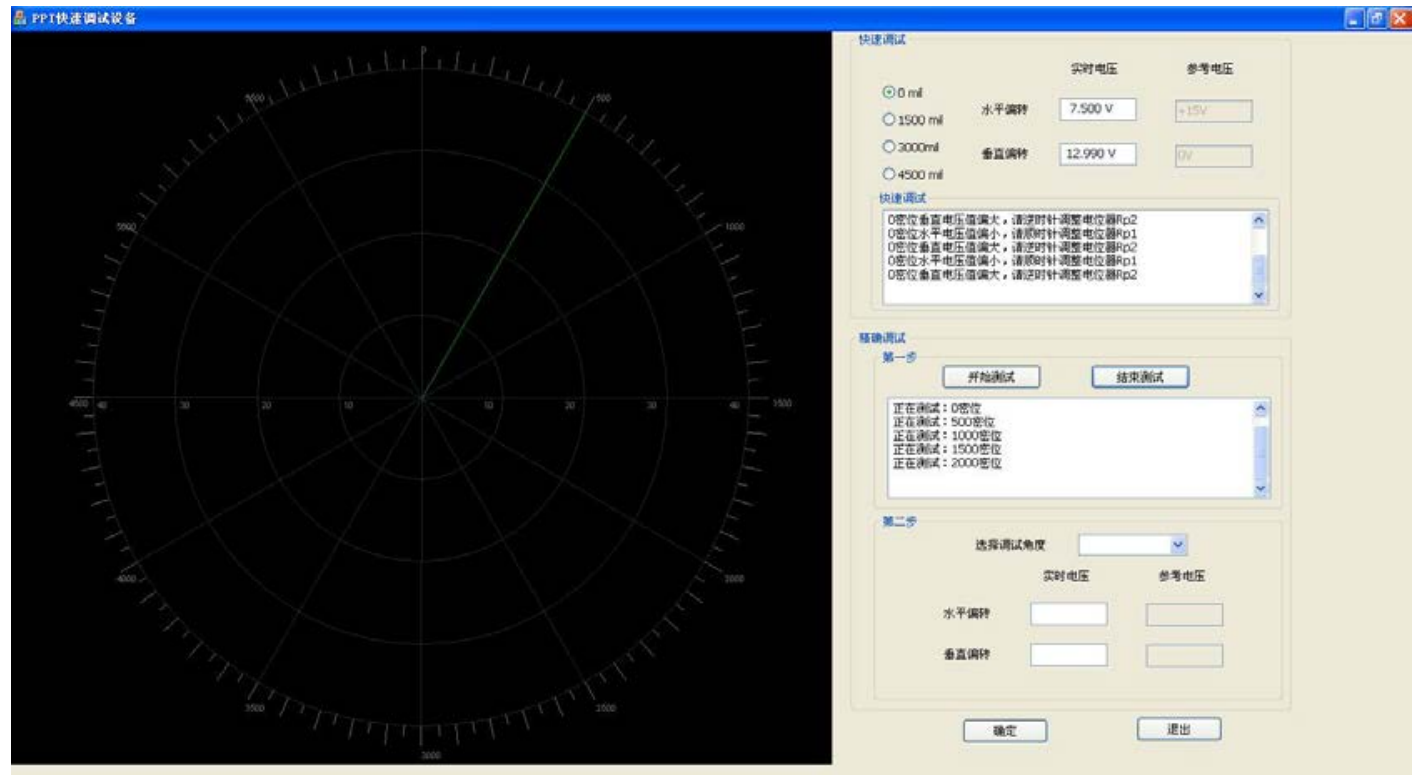

Figure 4 Interface of the software of the debugging device.

\section{Conclusion}

The radar PPI fast debugging device introduced in this paper can accomplish the measurement and analysis of radar PPI display control circuit signals, and provide a debugging plan. The original design objective is achieved. One difficult point of the design is to realize real-time and precise acquisition of the analog signals. After acquisition, the data are processed with the improved peak-to-peak value computation algorithm to get the precise peak-to-peak value. On this basis, the scanning line is plotted on the screen and a detailed debugging plan is obtained. As we can see, the debugging methods and devices of radar PPI will be more convenient and more efficient with the 
evolution of electrical techniques.

\section{References}

[1]Liu Guiying, Liu Yuanzhi, Chen Jiaqing. Design of the Airborne Warning Display Controller Based on FPGA. Aerospace Control[J].2005(5)

[2]FreundY, SehaPire R.E, A decision theoretic gene-realization of online learning and an Application to boosting. Journal of computer and system sei-enee [C],1997,55(l),119-139

[3]Alaa Eleyan, Huseyin Amanli. Adaptive and Fixed Eigenspace Methods with a Novel Fitness Measure for Video based Face Recognition, IEEE 2009.9:14-16

[4]Wang Gang, Zhou Ji Liu. Face attendance method research and realization on dynamic vedio [J], computer science ,2008(35):294-297 